\title{
Competenclas de la Estructura Directiva de las Organizaciones Sanitarlas. Sistema de Dirección, Gestión y Desarrollo de Recursos Humanos Basado en el Modelo de Gestión por Competencias
}

\author{
Francisco Fuentes Rodriguez \\ Hospital de Poniente. El Ejido
}

RESUMEN

El sistema de Gestión por Competencias (SGPC) se fundamenta principalmente en el desarrollo organizacional desde un prisma integrador de los objetivos perseguidos. siendo estos: la unificación de principios, formas de actuar, gestión de recursos bumanos, tecnologia, información, objetivos comunes, procesos y personas, sin descuidar aspectos tales como el propio entorno. los usuarios y el conjunto de normas y/o valores asociados, es decir, la cultura organizacional.

El presente articulo tiene como objetivo el desarrollo del denominado Mapa de Competencias de la Estructura Directiva de una Organización Sanitaria.

La metodologia que se ba seguido se fundamenta en paneles de expertos, se ban utilizado una serie de técnicas grupales para la elaboración de las diferentes competencias que confiere el puesto, tanto de caräcter técnico, como aquellas que comprenden las habilidades $y$ las actitudes necesarias para el desarrollo de un estilo de liderazgo basado u orientado tanto a resultados / tareas, como a las personas. Se ha elaborado un listado de las competencias necesarias para el puesto a partir de tres fases: la fase A o también llamada de selección, en la que a los aspirantes se les exige como condición imprescindible, si asi se ha requerido por el equipo de expertos que ha confeccionado y desarrollado las competencias del puesto; la fase $B$ o de experto, en la cual se les exige un mayor numero de competencias debido al grado de experticia que ba adquirido, y la fase $C$ o de excelencia, en la que se desarrolla un grado máximo de excelencia profesional debido al conocimiento profundo en las competencias a desarrollar. Estas fases en las que se vertebra el desarrollo de las competencias nos ofrece un instrumento potente que nos permite desarrollar planes formación y de desarrollo individuales, a la vez que sirve de elemento predictor y evaluador del 
desempeño profesional. Finalmente. se ponderan cada una de las competencias en función de la importancía y trascendencia de las competencias enumeradas en aras a establecer un perfil competencial de excelencia profesional.

Palabras clave: Recursos Humanos. Gestión por Competencias. Estructura Directiva, Habilidades, Actitudes, Desarrollo. Liderazgo. Organización.

ABstract

The System of Management of Competences (SGPC) is based mainly in the organization development from a point of lieu' integrating of the aims pursued, being these: the unifica-. cion of principles, forms of acting. management of human resources, technology, information, common objectives, processes and people, without neglecting aspects such as the own environment. the users and the associated set of norms and/or values, that is to say, the organizacional culture.

This article has like objective the development of the called map of competences of the directive structure of a sanitary organization.

Metbodology is based on paneling of experts and the procedure for its development, bas. been has based on groups techniques, in the elaboration of the different competences that the position confers, so much of technical character, as in the abilities and necessary attitudes for the development of a style of leadership based or oriented so much to results tasks, as to the people. A series of necessary competences designed in three phases is developed, the A phase or also called of selection, which the aspirants is required to them like essential condition. if has been required by the team of experts whom it bas made and developed the competences of the position. The $B$ phase of expert, in which is required them a greater number of competences due to the degree of experience that it bas acquired, and the $C$ phase or of excellence in which maximum of professional excellence due to the deep knowledge in the competences is developed to a degree to develop. These phases in which vertebran combine the development of the competences offer a powerful instrument to us so that, among others factors, we develop to individual plans of formation and development, like predicting and evaluate element of the functional professional. To complete of the analysis elements to consider each one of the competences in function of the importance and to establisb a profile of professional excellence.

Key words: Human Resources, Competences for Management, Directive Structure, Ability, Attitude, Development, Organization.

\section{INTRODUCCIÓN}

El desarrollo y gestión de los recursos humanos en las organizaciones actuales ha sufrido en los últimos años un profundo cambio, en gran medida mediatizado por parámetros económicos. Así, en la década de los sesenta en los que el entorno económico se caracterizaba fundamentalmente por un prisma cerrado orientado a la producción, la dirección de recursos humanos tiene unos claros tintes administrativos, como queda reflejado en la propia denominación de estas unidades: Administración de Personal. Por su parte, durante los años setenta y ochenta el entorno económico provoca una mayor apertura del mercado y la dirección de Recursos Humanos adopta una orientación más técnica. Pero am- 
bos sistemas de dirección sucumben en la última década ante la alta competitividad a la que se ven sometidas las empresas, al desarrollo de sistema de calidad. de innovación etc, sustentándose un nuevo prisma de gestión de recursos humanos basado en la estrategia de la dirección de equipos de trabajo. Por tanto. desde el enfoque estratégico de los recursos humanos, las personas pasan a copar el primer plano, pues son consideradas como la verdadera ventaja competitiva de las organizaciones. En este sentido, el desarrollo de cualquier organización actual viene mediatizado por la gestión y dirección del conocimiento, la capacidad de acumulación, y la renovación profesional mediante el aprendizaje y la formación continua.

Así pues, cada vez son más las organizaciones que de alguna manera tratan de optimizar la gestión de sus recursos humanos (RRHH), en tanto que determinadas políticas de gestión, planificación, gestión previsional, desempeño de superior categoría, o estilos de dirección e implicación organizacional, suponen una serie de ventajas tales como mayores cotas de eficiencia profesional, objetivización de los procesos, desarrollo y estimulación de competencias, o una motivación de los trabajadores, entre otras.

Ahora bien, toda organización está sujeta a una serie de cambios y necesidades que le impone su propio entorno y, en este sentido, las instituciones sanitarias como organizaciones "vivas" también se ven afectadas, siendo la sociedad, en este caso, quien actúa como motor de cambio; por ello necesitan dotarse, entre otros aspectos, de modelos de gestión profesional que optimicen los servicios que se les encomiendan y garanticen criterios de satisfacción no sólo al usuario que recibe atención sanitaria por parte de los profesionales que les atienden. sino que también éstos últimos puedan percibir que su trabajo está en consonancia con las exigencias del puesto y la capacidad percibida de éste para hacer frente a las demandas del puesto.

Consciente de ello, la Administración Pública, basándose en criterios de calidad, ha diseñado una política de gestión de sus recursos humanos orientada hacia estándares de gestión y desarrollo fundamentados en la optimización profesional y asistencial.

\section{¿Que Entendemos Por Competencias?}

Son numerosas las acepciones que aportan valor y contenido al concepto de competencia, si bien, el denominador común que determina su significado podría circunscribirse a las características personales que subyacen a los comportamientos. Así, los conocimientos, las habilidades, las formas de pensar o actuar, las actitudes, los valores o los rasgos de personalidad, determinan en buena medida el concepto de competencia. 
Autores clásicos como McClelland (1973), donde presenta las bases del enfoque basado en gestión por competencias, ha actuado como "cemento" y forjado para el desarrollo de todo un sistema de gestión basado en las citadas competencias. Sin duda, a partir de sus trabajos, han sido numerosos los autores, sobre todo en las dos últimas décadas, que han desarrollado un modelo de gestión basado en las competencias.

La propuesta presentada por Boyatzis (1982) se centra en las competencias que deben y tienen que desarrollar los miembros que componen la estructura directiva, haciendo referencia a las competencias nucleares o "competencias umbral" que deben poseer los dirigentes, y las denominadas "competencias superiores" que son aquellas que definen el perfil de excelencia profesional que determina un directivo considerado "excelente" respecto a otro que posee una actuación umbral. En tal sentido el autor define las competencias como "aquellas caracteristicas subyacentes de las personas, que estân causalmente relacionadas con un desempeño bueno o excelente en un puesto de trabajo concreto y en una organización concreta"

De los planteamientos de Boyartzis (1982) se desprende un conjunto de factores que acompañan a la definición de competencias: la motivación del trabajador, que en cualquier caso debe centrarse en los componentes intrínsecos de esta; los rasgos de carácter, tales como concepto de sí mismo, las actitudes o los valores que guíen su desempeño; una gran variedad de conocimientos de carácter técnico; y las habilidades o capacidades cognoscitivas y de comportamiento.

Las competencias no son un conjunto de elementos aislados de análisis y desarrollo individual sino más bien todo lo contrario, en cuanto que actúan como. componentes interrelacionados y que se desarrollan de forma conjunta, en todas las funciones del desempeño del trabajo (Le Bofert, Barzucchetti y Viccent,1993).

También las propias Administraciones, tanto nacionales como supranacionales; han definido el concepto de competencia. En España se han desarrollado decretos en los que se define el certificado de competencia. En este sentido, el Real Decreto: 1396/1995, del Ministerio de la Presidencia dentro del marco de la Directiva Europea 92/51/CEE define competencia como "un título que sanciona una formación y se expide a raíz de una valoración de las cualidades personales, de las aptitudes: o de los conocimientos del solicitante, considerados como fundamentales para el ejercicio de una profesión." (Pp.75)

La concepción del término competencia genera muchas veces un carácter vàriable en función de las personas que lo utilicen (Levy-Levoyer, 1997), si bien dicha concepción se ha extendido cada vez más en la gestión estratégica que lás empresas utilizan como sistemas de gestión basados en competencias (Pereda y Berrocal, 2001). 
Por tanto, observamos que las competencias presentan un doble prisma, por un lado, están aquellas competencias observables y por otro las denominadas intangibles que componen el mayor porcentaje de la variable competencia. Así, las actitudes, los rasgos de personalidad, los valores o los componentes motivacionales, entre otros, conforman la estructura que sustentan los componentes de las competencias.

¿En Que Se Basa la Gestion Por Competencias?

La gestión basada en las competencias se vertebra, fundamentalmente, en la calidad y disposición del equipo humano que compone una determinada organización, que a su vez va a repercutir en la integración y desarrollo de sus miembros, así como en las cualidades de sus integrantes, entendidas éstas, como elementos potenciales y diferenciadores de unos miembros respecto a otros.

Así pues, las listas de competencias que más adelante definimos en el denominado Mapa de Competencias, son las que determinarán un estilo de dirección centrado en el factor humano principalmente, esto es, un liderazgo centrado u orientado hacia las relaciones de sus miembros, presentando a su vez valores más colectivistas. Descle la implicación de la dirección en un sistema de gestión por competencias (SGPC) se desarrollarán las funciones de una manera sistemática y coordinada, de forma tal que puedan cumplirse los objetivos que se persiguen en este sistema de gestión:

- Implantación desde la estructura directiva de una cultura basada en la excelencia profesional.

- Toma de decisiones de forma objetiva y con criterios homogéneos.

- Desarrollo de la carrera profesional.

- Incorporar en cada proceso definido la gestión integral.

- Mejora continua y calidad total.

- Proceso y feedback de las políticas implantadas.

En definitiva, el fin perseguido es la visión integral del equipo hacia la consecución de los objetivos organizacionales.

Este enfoque estratégico de recursos humanos centrado en el SGPC, nos permite actuar en todos los niveles del proceso de gestión que va desde la selección, los planes de desarrollo profesional, la evaluación del desempeño, la rotación de puestos, y en general todos los procesos asociados a la gestión de los recursos humanos. 


\section{Componentes De Las C_ompetencias}

Es difícil delimitar qué componentes de las competencias son los necesarios para' un buen desempeño del trabajo, así como analizar y desarrollar el tipo de comportamiento que se considera adecuado para cada puesto, pero como señalan Pereda y Berrocal (2001), podemos utilizar la evaluación del desempeño como instrumento para delimitar dichos comportamientos. De igual manera podemos identificar los puntos fuertes y débiles de los profesionales, ayudándoles desde esta perspectiva a la estimulación y desarrollo de los componentes esenciales.

Las competencias entendidas, como el hacer o el desempeño en las funciones exigidas por el puesto, llevan consigo aparejadas una serie de elementos o variables; tales como los conocimientos (saber), las capacidades o habilidades (saber bacer), las actitudes e intereses (saber estar), la motivación (querer hacer), así como las aptitudes y rasgos de una parte y los recursos materiales y humanos de otra (poder hacer).

Al hablar de los componentes de las competencias nos centramos fundamental-: mente en tres variables:

Los conocimientos: Hacen referencia a la adquisición de todos aquellos conceptos de carácter técnico y/o social que el puesto requiere y que normalmente se adquieren en le proceso de enseñanza-aprendizaje, a partir de la aplicación de una metodología y técnicas específicas en función del contenido a aprender.

Las babilidades: Son un conjunto de capacidades ó destrezas que se adquieren durante el desempeño profesional, y que, serán tanto mejores en la medida en que se complementen con los conocimientos.

Las actitudes: Se refieren a los componentes intangibles de las competencias, es decir, a un conjunto de rasgos de carácter, normas y valores, creencias, etc. Aspectos todos ellos que si bien son singulares, suponen un factor esencial en el desempeño de las funciones del puesto.

\section{Metodología Y Desarrollo Del Mapa De Competenclas De la Estructura Directiva}

Como vimos más arriba, el objetivo fundamental del sistema de Gestión por Competencias es dirigir de manera integral los Recursos Humanos dentro de las organizaciones, a través del aprovechamiento de los conocimientos, habilidades 0 destrezas y actitudes de los miembros que las componen.

Mediante un sistema de gestión por competencias se trata de analizar y descubrir al profesional que desarrolla con éxito sus funciones en un puestọ, así como de analizar sus habilidades, conocimientos y actitudes, lo que nos permitirá poder utilizar estrategias de mantenimiento de estos profesionales a fin de que se integren y permanezcan en la organización, pues hay estudios que señalan que el $40 \%$ de las nuevas contrataciones de directivos fracasan en los primeros 18 meses y que menos 
del $9 \%$ de los directivos que fracasan lo hacen por incapacidad técnica. Incluso consultoras como Hay Group, manifiestan que "el $70 \%$ de las personas que abandonan una organización lo hacen por una mala relación con su superior". Es por ello por lo que diferentes autores señalan que el liderazgo debe fundamentarse en la Llamada inteligencia emocional, entendida ésta como la capacidad de gestionarse a sí mismo y de relacionarse con los demás de forma fructífera (Estrada, 2000).

En nuestro estudio nos centraremos en la Estructura Directiva de una organización sanitaria, elaborando una Mapa de Competencias que nos permita adaptar y cohesionar todos los procesos de Recursos Humanos al modelo de competencias a través de una Gestión Integral de Recursos Humanos.

Ahora bien, no hemos de olvidar que para que la implantación de este enfoque de gestión de recursos humanos sea posible se requiere una dirección que integre un proceso de visión global y completo de empresa, que sea capaz de integrar adecuadamente los equipos de trabajo y de dirigirlos hacia el logro de los objetivos planteados en función de los retos y de las oportunidades del entorno, centrándose en cualquier caso en los criterios de excelencia profesional y teniendo como meta, en nuestro caso, las políticas de calidad organizacional definidas por la Consejería de Salud de la Junta de Andalucía, y siempre la referencia de que "el ciudadano es el eje del sistema".

Como veremos más adelante en detalle, hemos desarrollado un primer marco de Competencias en donde se integran las denominadas competencias Nucleares o centrales de la estructura directiva, dejando para un segundo paso el desarrollo del resto de competencias así como la consideración de idoneidad y deseabilidad profesional de cada una de ellas en las tres fases que seguimos: fase A ó de selección, fase B ó experto y fase C ó excelente. También se concretarán los perfiles de excelencia profesional de cada una de las competencias descritas en el mapa de competencias.

Con la elaboración del citado Mapa de Competencias pretendemos unos objetivos bien definidos:

- Identificar los conocimientos, habilidades y actitudes que han de poseer los profesionales que desempeñan puestos directivos.

- Sensibilizar, hacer participar y motivar a un conjunto de profesionales para que aporten conocimiento, experiencia y valor añadido a esta estrategia de gestión por competencias.

- Coordinar al grupo de expertos en tareas de dirección, desarrollo y gestión, facilitando recursos, dinámicas de grupo y herramientas metodológicas que maximicen su rendimiento y eficacia.

- Fomentar la relación entre los directivos y los empleados a través de la formación, la fidelización, mejora del clima laboral y en la obtención de beneficios tangibles o intangibles por ambas partes (Baltanás, 1999).

- Proporcionar retroinformación de calidad y constructiva, (Slivinscki y Bourgeois, 1977), a todos los niveles de la organización. 
Para el análisis y la definición marco del Mapa de Competencias de la estructura direc:tiva, hemos seguido una metodología cualitativa, utilizando el procedimiento de panel de expertos.

Contamos con un panel de expertos de un carácter multidisciplinar, compues: to por 10 miembros: de una parte, 6 directivos de diferentes áreas, tanto de estructuras asistenciales como de estructuras no asistenciales, y de otra parte, 4 empleados con un alto nivel de conocimientos sobre diversos puestos de trabajo de la organización.

Pero también, hemos de destacar la figura del moderador/coordinador en el proceso: de definición de competencias, cuya función principal estriba en conseguir el consensọ, acuerdo así como delimitar las funciones y competencias a enmarcar en el llamado mapa de competencias.

\section{Desarrollo de la Técnca}

Una vez definidos los objetivos, constituido el panel de expertos y nombrado el moderador/coordinador, la definición del Mapa de Competencias se desarrolla en tres sesiones:

$1^{\text {a }}$ Sesión: Básicamente teórica, donde el coordinador del taller explica los contenidos. teóricos a desarrollar basado en el Sistema de Gestión por Competencias, los objetivos a conseguir, así como la metodología de trabajo.

$2^{2}$ Sesión: El objetivo de esta sesión del panel es la generación espontánea, interactiva y múltiple de las competencias profesionales necesarias de la estructura directiva, sin generar miedos o reservas a la participación. A tal efecto se utilizan técnicas de carácter grupal: Phillips 6/6, Brainstorming, Rol Playing, etc.

Con la información generada en esta sesión, el coordinador del panel trabajará las competencias obtenidas, identificará los conceptos e integrará las competencias definidas en el mapa de competencias.

A partir de este proceso de análisis competencial se extraen las competencias fundamentales o centrales del puesto perteneciente a la estructura directiva y las competencias secundarias o complementarias necesarias a desarrollar en las fases de experticia y excelencia, a través de los planes de desarrollo individuales.

$3^{a}$ Sesión: el moderador del panel presenta por escrito a todos los miembros las competencias generadas en la segunda sesión. Las competencias de conocimiento, habilidad y actitud se presentan con las tres fases de incorporación, madurez y excelencia, asignándole a cada competencia y en cada una de las fases la inicial (I) de imprescindible o (D) de deseable, según los criterios de los expertos. Asimismo, los miembros del panel han de establecer un orden de prioridad de las competencias en una escala de 1 a 4 : puntos, según el nivel que consideran que la misma debe concurrir en el profesional para. el proceso que nos ocupa. 


\section{Mapa de Competencias:}

\section{Estructura Directiva}

\begin{tabular}{|c|c|c|c|c|c|c|c|c|c|}
\hline $\begin{array}{l}\text { MACRO- } \\
\text { COMPETENCIAS }\end{array}$ & $\begin{array}{l}\text { COMPETENCIAS } \\
\text { ASOCLADAS }\end{array}$ & $\begin{array}{c}\text { DESCRIPCIÓN } \\
\text { DEL COMPORTAMIENTO }\end{array}$ & 4 & $\infty$ & 0 & & $\mathrm{ER}$ & EN & \\
\hline & & & \& & $\lesssim$ & 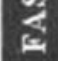 & & 2 & 3 & 4 \\
\hline \multirow{3}{*}{$\begin{array}{l}\text { GESTIÓN DEL } \\
\text { CAMBIO }\end{array}$} & Iniciativa & 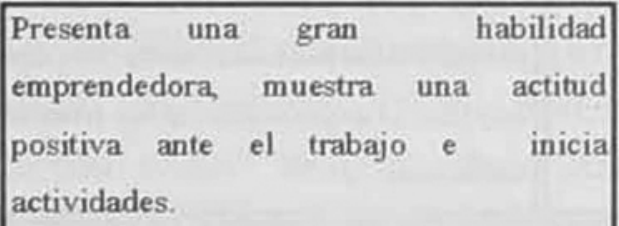 & D & I & I & & & & \\
\hline & \begin{tabular}{|l} 
Asunción de \\
Riesgos
\end{tabular} & $\begin{array}{l}\text { Capacidad de afrontar nuevos retos, } \\
\text { desafia prácticas }\end{array}$ & D & I & I & & & & \\
\hline & $\begin{array}{l}\text { Flexibilidad } \\
\text { Ad aptabilidad }\end{array}$ & $\begin{array}{l}\text { Se acomoda fácilmente a las resoluciones } \\
\text { propuestas, se adapta con rapidez al } \\
\text { cambio. }\end{array}$ & I & I & I & & & & \\
\hline \multirow{3}{*}{$\begin{array}{l}\text { PLANIFICACIÓN } \\
\text { ORGANIZACIÓN }\end{array}$} & $\begin{array}{l}\text { Pensamiento } \\
\text { Analitico }\end{array}$ & $\begin{array}{l}\text { Capacidad de para distinguir y separar las } \\
\text { partes de un todo hasta llegar a conocer } \\
\text { sus principios o factores que los } \\
\text { componen, desarrollando juicios } \\
\text { razonables y decisiones logicas. }\end{array}$ & I & I & I & & & & \\
\hline & Planificación & $\begin{array}{l}\text { Capacidad para someter a un plan } \\
\text { estudiado una actividad o proceso. }\end{array}$ & I & $\sqrt{I}$ & $\sqrt{\mathbf{I}}$ & & & & \\
\hline & $\begin{array}{l}\text { Orientación a la } \\
\text { Calidad }\end{array}$ & $\begin{array}{l}\text { Orientación hacia la consecución de los } \\
\text { objetivos organizacionales, mediante la } \\
\text { estrategias de satisfacción tanto al cliente } \\
\text { interno como al usuario del producto o } \\
\text { servicio. }\end{array}$ & I & I & I & & & & \\
\hline
\end{tabular}




\begin{tabular}{|c|c|c|c|c|c|c|c|c|c|}
\hline \multirow[t]{2}{*}{$\begin{array}{l}\text { MACRO- } \\
\text { COMPETENCIAS }\end{array}$} & \multirow[t]{2}{*}{$\begin{array}{c}\text { COMPETENCIAS } \\
\text { ASOCLADAS }\end{array}$} & \multirow[t]{2}{*}{$\begin{array}{c}\text { DESCRIPCIÓN } \\
\text { DEL COMPORT AMIENTO }\end{array}$} & \multirow{2}{*}{$\sum_{2}^{4}$} & \multirow{2}{*}{$\sum_{\infty}^{\infty}$} & \multirow{2}{*}{$\begin{array}{l}0 \\
\frac{6}{5} \\
\frac{5}{2}\end{array}$} & \multicolumn{4}{|c|}{$\begin{array}{c}\text { PERFIL DE } \\
\text { EXCELENCEA }\end{array}$} \\
\hline & & & & & & & 2 & 3 & 4 \\
\hline \multirow{4}{*}{$\begin{array}{l}\text { DESTREZAS } \\
\text { INTER } \\
\text { PERSONALES }\end{array}$} & Comunicación Oral & $\begin{array}{l}\text { Capacidad para transmitir información, } \\
\text { expresa los puntos de vista con claridad. }\end{array}$ & $\bar{D}$ & I & I & & & & \\
\hline & Sensibilidad & $\begin{array}{l}\text { Manifiesto, patente al entendimiento, } \\
\text { sensible a las necesidades de las personas; } \\
\text { implica a las personas en los planes y las } \\
\text { decisiones }\end{array}$ & I & I & I & & & & \\
\hline & Relaciones & $\begin{array}{l}\text { Potencia y desarrolla las relaciones de } \\
\text { trabajo, conecta con rapidez. }\end{array}$ & D & $\mathrm{D}$ & $I$ & & & & \\
\hline & Trabajo en Equipo & $\begin{array}{l}\text { Habilidad para desarrollar e integrar } \\
\text { ideas, proyectos y objetivos comunes. }\end{array}$ & D & I & I & & & & \\
\hline \multirow{4}{*}{$\begin{array}{l}\text { ORIENTACIÓN } \\
\text { A } \\
\text { RESULTADOS }\end{array}$} & Logro & $\begin{array}{l}\text { Llevar a los proyectos o ideas hacia la } \\
\text { excelencia, dispuesto a avanzar. }\end{array}$ & I & I & I & & & & \\
\hline & \begin{tabular}{lll|} 
Centrado en el \\
usuario
\end{tabular} & $\begin{array}{l}\text { Orientado hacia las demandas del usuario, } \\
\text { vive el servicio al cliente, se centra en la } \\
\text { calidad. }\end{array}$ & I & I & $\overline{\mathrm{I}}$ & & & & \\
\hline & $\begin{array}{l}\text { Conciencia de } \\
\text { organización }\end{array}$ & $\begin{array}{l}\text { Objetivo, realista, controla los costes, se } \\
\text { fija metas amplias. }\end{array}$ & D & I & I & & & & \\
\hline & $\begin{array}{ll}\text { Orientación al } \\
\text { Aprendizaje }\end{array}$ & $\begin{array}{l}\text { Actitud de mejora y aprendizaje continua } \\
\text { sobre la proyección de su trabajo. }\end{array}$ & D & I & I & & & & \\
\hline \multirow{4}{*}{ LIDERAZGO } & $\begin{array}{l}\text { Autoridad } \\
\text { Presencia }\end{array}$ & $\begin{array}{l}\text { Crédito que un profesional posee en su } \\
\text { actuación profesional, así como la } \\
\text { ejecución de un liderazgo participativo. }\end{array}$ & D & I & I & & & & \\
\hline & $\begin{array}{l}\text { Motivación a los } \\
\text { demás }\end{array}$ & $\begin{array}{l}\text { Proporciona confianza, da autonomia, } \\
\text { responsable y motiva a los miembros del } \\
\text { equipo, delega las tareas con eficiencia. }\end{array}$ & D & I & $I$ & & & & \\
\hline & $\begin{array}{l}\text { Desarrollo } \\
\text { Profesional }\end{array}$ & $\begin{array}{l}\text { Actitud y potencial para desarrollar } \\
\text { nuevos retos, centrándose en la habilidad } \\
\text { de comunicación. }\end{array}$ & D & I & I & & & & \\
\hline & Resistencia & $\begin{array}{l}\text { Actitud de afrontamiento con eficiencia } \\
\text { hacia las presiones y demandas en su } \\
\text { actuación profesional. }\end{array}$ & D & $\mathrm{D}$ & I & & & & \\
\hline
\end{tabular}




\section{CONCLUSIONES:}

En el presente artículo hemos tratado de ofrecer el desarrollo del Mapa de Competencias en cuanto a conocimientos, habilidades y actitudes necesarias a poseer por la Estructura Directiva. Se ofrece una marco de referencia con el objetivo de delimitar aquellos elementos favorecedores de la excelencia directiva, así como el liderazgo que estos deben implantar en las organizaciones. Pero sin duda, el enfoque estratégico quedará completo en la medida en que establezcamos sistemas de auditoria permanentes o continuos, que nos ofrezcan feedback sobre las políticas implementadas, programas, prácticas, así como el desarrollo de la eficiencia y eficacia profesional. Dicho sistema de auditoria cumple una doble función: por una parte, actúa como sistema de información directiva, es decir, permite conocer el estado objetivo, posibilitando así el desarrollo de procesos de gestión y/o desarrollo de Recursos Humanos, y por otra parte, actúa igualmente como sistema de control y evaluación de la implementación de las políticas y procesos predefinidos.

El entendimiento respecto a la dirección y gestión los recursos humanos pasa por la visión integral, en cuanto a los componentes humanos y las actividades de la clirección, deduciendo las políticas a desarrollar, los objetivos y los planes estratégicos de la organización (Pereda y Berrocal, 2001). El enfoque de las competencias quedará validado en la medida que se establezcan los objetivos precisos, la metodología de trabajo y la evaluación continuada de la actuación profesional.

Besseyre des Hots (1989) señalan que la función estratégica debe dirigirse a tres dimensiones esenciales para la aplicación de la estrategia de la empresa, a saber, la adquisición, la estimulación y el desarrollo de las competencias, como verdadera línea política de gestión de profesionales que debe implantarse desde la dirección. En la adquisición de las competencias es necesario analizar y auditar cómo se facilita o proporciona a la organización las competencias necesarias. Desde la estimulación de competencias se analizan y/o auditan cómo se potencian y motivan las competencias latentes para alcanzar los objetivos, y desde el marco del desarrollo de las competencias, se trata de analizar / auditar cómo evolucionan las competencias para adecuarse a los cambios estratégicos.

Este marco posibilita una visión completa e integradora de la realidad sociolaboral, la cual pone de manifiesto la variedad de tareas y el valor estratégico de la Dirección de Recursos Humanos.

\section{Bibliografia}

Baltanás J. (1999). Mentoring.-Training and Development Digest. Enero, 14, 44-46. Besseyre des Horts, C (1989). Gestión Estratégica de los Recursos Humanos. Bilbao. Deusto.

Boyatzis, R.E. (1982). The competent manager: A model for effective performance. Nueva York. John Wiley \& Sons. 
Estrada A. 2000. Claves para gestionar las empresas. Comunicación presentada a Congreso de Directivos. Madrid, Septiembre de 2000.

Le Bofert, G., Barzucchetti, S. y Viccent, F. (1993). Cómo Gestionar la Calidad de h, Formación. Barcelona: Gestión 2000.

Levy-Levoyer, C.(1997). Gestión de las competencias. Barcelona: Gestión 2000.

McClelland. D.C. (1973). Testing for competence rather than for intelligence. Ameri can Psychologist, 28, 1-14

Pereda S, y Berrocal F, (2001). Gestión de Recursos Humanos por Competencias Madrid. Centro de Estudios Ramón Areces.

Pereda, S. y Berrocal, F. (2001). Técnicas de Gestión de Recursos Humanos por Competencias. Madrid. Centro de Estudios Ramón Areces.

Prieto, J.M. (1977). Prólogo. En C. Levy-Leboyer. Gestión de las competencias. Barcelona. Gestión 2000.

Slivinski, L. W. y Bourgeois, R.P. (1977). Feedback of assessment center results. En J.L. Moses y W. C. Byham. Applying the assessment center meted. New York Pergamom Press. 143-159. 